\title{
Polynomial Generalizations and Combinatorial Interpretations for Sequences Including the Fibonacci and Pell Numbers
}

\author{
Cecília Pereira de Andrade, José Plínio de Oliveira Santos*, \\ Elen Viviani Pereira da Silva, Kênia Cristina Pereira Silva \\ Universidade Estadual de Campinas, Campinas-SP, São Paulo, Brazil \\ Email: cissamat@gmail.com, josepli@ime.unicamp.br, elenvps@gmail.com,keycpsilva@gmail.com
}

Received September 12, 2012; revised October 12, 2012; accepted October 22, 2012

\begin{abstract}
In this paper we present combinatorial interpretations and polynomials generalizations for sequences including the Fibonacci numbers, the Pell numbers and the Jacobsthal numbers in terms of partitions. It is important to mention that results of this nature were given by Santos and Ivkovic in two papers published on the Fibonacci Quarterly, Polynomial generalizations of the Pell sequence and the Fibonacci sequence [1] and Fibonacci Numbers and Partitions [2], and one, by Santos, on Discrete Mathematics, On the Combinatorics of Polynomial generalizations of Rogers-Ramanujan Type Identities [3]. By these results one can see that from the q-series identities important combinatorial information can be obtained by a careful study of the two variable function introduced by Andrews in Combinatorics and Ramanujan's lost notebook [4].
\end{abstract}

Keywords: Partitions; Fibonacci Numbers; Pell Numbers; Jacobsthal Numbers; q-Analog

\section{Introduction}

In this paper following some ideas introduced by Andrews [4] and results given by Santos [5] we give polynomial generalizations and combinatorial interpretations for sequences, including Fibonacci numbers, the Pell numbers and the Jacobsthal numbers in terms of partitions. To do this we use identities $12,16,20,28,44,66$, 67, 80 and 81, listed below, that are among the 130 q-series identities given by Slater in [6].

$$
\begin{aligned}
& \sum_{n=0}^{\infty} \frac{(-1 ; q)_{n} q^{\left(\begin{array}{c}
n+1 \\
2
\end{array}\right)}}{(q ; q)_{n}} \\
& =\frac{(-q ; q)_{\infty}}{(q ; q)_{\infty}}\left(q^{4} ; q^{4}\right)_{\infty}\left(q^{2} ; q^{4}\right)_{\infty}^{2} . \\
& \sum_{n=0}^{\infty} \frac{q^{n^{2}+2 n}}{\left(q^{4} ; q^{4}\right)_{n}} \\
& =\frac{\left(-q ; q^{2}\right)_{\infty}}{\left(q^{2} ; q^{2}\right)_{\infty}}\left(-q^{5} ; q^{5}\right)_{\infty}\left(q^{4} ; q^{5}\right)_{\infty}\left(q^{1} ; q^{5}\right)_{\infty} .
\end{aligned}
$$

*Partially supported by FAPESP (Fundação de Amparo à Pesquisa do Estado de São Paulo).

$$
\begin{aligned}
& \sum_{n=0}^{\infty} \frac{q^{n^{2}}}{\left(q^{4} ; q^{4}\right)_{n}} \\
& =\frac{\left(-q ; q^{2}\right)_{\infty}}{\left(q^{2} ; q^{2}\right)_{\infty}}\left(-q^{5} ; q^{5}\right)_{\infty}\left(q^{3} ; q^{5}\right)_{\infty}\left(q^{2} ; q^{5}\right)_{\infty} . \\
& \sum_{n=0}^{\infty} \frac{\left(-q^{2} ; q^{2}\right)_{n} q^{n^{2}+n}}{(q ; q)_{2 n+1}} \\
& =\frac{\left(-q ; q^{2}\right)_{\infty}}{\left(q ; q^{2}\right)_{\infty}}\left(-q^{6} ; q^{6}\right)_{\infty}\left(-q^{5} ; q^{6}\right)_{\infty}\left(-q^{1} ; q^{6}\right)_{\infty} . \\
& \sum_{n=0}^{\infty} \frac{q^{3} \frac{3}{2 n}(n+1)}{\left(q ; q^{2}\right)_{n+1}(q ; q)_{n}} \\
& =\frac{1}{(q ; q)_{\infty}}\left(q^{10} ; q^{10}\right)_{\infty}\left(q^{8} ; q^{10}\right)_{\infty}\left(q^{2} ; q^{10}\right)_{\infty} . \\
& \sum_{n=0}^{\infty} \frac{\left(-1 ; q^{4}\right)_{n}\left(-q ; q^{2}\right)_{n} q^{n^{2}}}{\left(q^{2} ; q^{2}\right)_{2 n}} \\
& =\frac{\left(-q ; q^{2}\right)_{\infty}}{\left(q^{2} ; q^{2}\right)_{\infty}}\left(q^{16} ; q^{16}\right)_{\infty}\left(q^{10} ; q^{16}\right)_{\infty}\left(q^{6} ; q^{16}\right)_{\infty} . \\
& +q\left(\left(q^{16} ; q^{16}\right)_{\infty}\left(q^{14} ; q^{16}\right)_{\infty}\left(q^{2} ; q^{16}\right)_{\infty}\right) .
\end{aligned}
$$




$$
\begin{aligned}
& \sum_{n=0}^{\infty} \frac{\left(-1 ; q^{4}\right)_{n}\left(-q ; q^{2}\right)_{n} q^{n^{2}+2 n}}{\left(q^{2} ; q^{2}\right)_{2 n}} \\
& =\frac{\left(-q ; q^{2}\right)_{\infty}}{\left(q^{2} ; q^{2}\right)_{\infty}}\left(\left(q^{16} ; q^{16}\right)_{\infty}\left(q^{10} ; q^{16}\right)_{\infty}\left(q^{6} ; q^{16}\right)_{\infty}\right. \\
& \left.-q\left(q^{16} ; q^{16}\right)_{\infty}\left(q^{14} ; q^{16}\right)_{\infty}\left(q^{2} ; q^{16}\right)_{\infty}\right) . \\
& \sum_{n=0}^{\infty} \frac{(-q ; q)_{n} q^{\frac{n(n+1)}{2}}}{\left(q^{2} ; q^{2}\right)_{n}\left(q ; q^{2}\right)_{n+1}} \\
& =\frac{(-q ; q)_{\infty}}{(q ; q)_{\infty}}\left(q^{21} ; q^{21}\right)_{\infty}\left(-q^{13} ; q^{21}\right)_{\infty}\left(-q^{8} ; q^{21}\right)_{\infty} \\
& -q^{2}\left(q^{21} ; q^{21}\right)_{\infty}\left(-q^{20} ; q^{21}\right)_{\infty}\left(-q ; q^{21}\right)_{\infty} . \\
& \sum_{n=0}^{\infty} \frac{(-q ; q)_{n} q^{\frac{n(n+1)}{2}}}{\left(q^{2} ; q^{2}\right)_{n}\left(q ; q^{2}\right)_{n}} \\
& =\frac{(-q ; q)_{\infty}}{(q ; q)_{\infty}}\left(q^{21} ; q^{21}\right)_{\infty}\left(-q^{11} ; q^{21}\right)_{\infty}\left(-q^{10} ; q^{21}\right)_{\infty} \\
& -q\left(q^{21} ; q^{21}\right)_{\infty}\left(-q^{17} ; q^{21}\right)_{\infty}\left(-q^{4} ; q^{21}\right)_{\infty} .
\end{aligned}
$$

Here we are using the standard notation of $q$-series

$$
\begin{aligned}
& (a)_{n}=(a ; q)_{n}=\prod_{j=0}^{\infty} \frac{\left(1-a q^{j}\right)}{\left(1-a q^{j+n}\right)} \\
& =(1-a)(1-a q) \cdots\left(1-a q^{n-1}\right) .
\end{aligned}
$$

when $n$ is a positive integer, and

$$
(a ; q)_{\infty}=(a)_{\infty}=\prod_{j=0}^{\infty}\left(1-a q^{j}\right) .
$$

\section{Background}

Before explaining how to get the combinatorial interpretation for the sequences mentioned above we need a few definitions.

Definition 2.1 The Pell numbers 1, 2, 5, 12, 29, $\cdots$, defined by the recurrence relation $p_{0}=1 ; \quad p_{1}=2$; $p_{n}=2 p_{n-1}+p_{n-2}, n \geq 2$ are the denominators of the sequence of rational numbers

$$
\frac{1}{1}, \frac{3}{2}, \frac{7}{5}, \frac{17}{12}, \frac{41}{29}, \frac{99}{70}, \cdots
$$

that are the continued fraction convergent to $\sqrt{2}$.

Definition 2.2 The Fibonacci numbers $0,1,1,2,3,5$, $8,13, \cdots$, are defined by

$$
F_{0}=0 ; \quad F_{1}=1 ; \quad F_{n}=F_{n-1}+F_{n-2}, n \geq 2 .
$$

Definition 2.3 The Jacobsthal numbers 1, 1, 3, 5, 11,
$21,43, \cdots$ are defined by

$$
J_{0}=1 ; \quad J_{1}=1 ; \quad J_{n}=J_{n-1}+2 J_{n-2}, n \geq 2 .
$$

Definition 2.4 The Gaussian polynomials are defined as follows:

$$
\left[\begin{array}{c}
n \\
m
\end{array}\right]_{q}= \begin{cases}\frac{(q)_{n}}{(q)_{m}(q)_{n-m}}, & 0 \leq m \leq n \\
0, & \text { otherwise. }\end{cases}
$$

Definition 2.5 The coefficients of $x^{j}$ in the expanded form of $\left(1+x+x^{2}\right)^{n}$ are given by:

$$
\left(\begin{array}{l}
n \\
j
\end{array}\right)_{2}=\sum_{h \geq 0}(-1)^{h}\left(\begin{array}{l}
n \\
h
\end{array}\right)\left(\begin{array}{c}
2 n-2 j \\
n-j-h
\end{array}\right) .
$$

We call these numbers trinomial coefficients.

The following two expressions are $q$-analog for the trinomial coefficients.

$$
\begin{aligned}
& T_{0}(m, A, q)=\sum_{j \geq 0}(-1)^{j}\left[\begin{array}{c}
m \\
j
\end{array}\right]_{q^{2}}\left[\begin{array}{c}
2 m-2 j \\
m-A-j
\end{array}\right] . \\
& T_{1}(m, A, q)=\sum_{j \geq 0}(-q)^{j}\left[\begin{array}{c}
m \\
j
\end{array}\right]_{q^{2}}\left[\begin{array}{c}
2 m-2 j \\
m-A-j
\end{array}\right] .
\end{aligned}
$$

Finally we define:

\section{Definition 2.6}

$$
\begin{aligned}
U(m, A) & =T_{0}(m, A, q)+T_{0}(m, A+1, q) . \\
C T(m, A) & =T_{1}\left(m, A, q^{1 / 2}\right) .
\end{aligned}
$$

\section{The Results from Equations (12) and (16)}

In [1-3] we have presented a number of results of the same nature as the ones given is this paper. For this reason we are not describing all the steps needed in the process but just a general description. In a series of two papers [6,7], Slater gave a list of 130 identities of the Rogers-Ramanujan type. In [4] Andrews introduced a two variable function in order to look for combinatorial interpretations for those identities. In [5] one of us, Santos, gave conjectures for explicit formulas for families of polynomials that can be obtained using Andrews' method for more than 70 identities of Slater's list. The conjectures listed there can be proved as we did in [3] for identity 20 , by doing a lot of calculations, or with the help of packages given by Sills in [8].

Then, following Andrews [4] one can introduce a parameter $t$ in the left hand side of (1) to get

$$
f_{12}(q, t)=\sum_{n=0}^{\infty} \frac{(-t ; q)_{n} t^{n} q^{\left(\begin{array}{c}
n+1 \\
2
\end{array}\right)}}{(t ; q)_{n+1}},
$$

from which a functional equation can be obtained: 


$$
(1-t) f_{12}(q, t)=1+(1+t) t q f_{12}(q, t q) .
$$

Knowing that the coefficient of $t^{n}$ in the expansion of (18) is a polynomial in q, i.e., that

$$
f_{12}(q, t)=\sum_{n=0}^{\infty} P_{n}(q) t^{n},
$$

it is easy to see that

$$
\begin{aligned}
& P_{0}(q)=1 ; P_{1}(q)=1+q ; \\
& P_{n}(q)=\left(1+q^{n}\right) P_{n-1}(q)+q^{n-1} P_{n-2}(q) .
\end{aligned}
$$

Santos gave in [5] the following conjecture for an explicity formula for this family of polynomials:

$$
\begin{aligned}
P_{n}(q) & =\sum_{j=-\infty}^{\infty} q^{8 j^{2}} C T(n, 1+8 j) \\
& -\sum_{j=-\infty}^{\infty} q^{8 j^{2}-8 j+2} C T(n, 3-8 j),
\end{aligned}
$$

where $C T(n, m)$, defined by Equation (17), is a $q$-ana$\log$ of the trinomial coefficient in the same way that the Gaussian polynomial is a $q$-analog of the binomial coefficient, that is, its limit, when $q$ approaches 1 , is equal to the trinomial coefficient given by (13).

To explain how to get a combinatorial interpretation for (18) we write it in the following form:

$$
\begin{aligned}
& \sum_{n=0}^{\infty} \frac{(-t ; q)_{n} t^{n} q^{\left(\begin{array}{c}
n+1 \\
2
\end{array}\right)}}{(t ; q)_{n+1}} \\
& =\frac{1}{1-t} \sum_{n=0}^{\infty} \frac{(1+t)(1+t q) \cdots\left(1+t q^{n-1}\right) t^{n} q^{\frac{n^{2}+n}{2}}}{(1-t q)\left(1-t q^{2}\right) \cdots\left(1-t q^{n}\right)} .
\end{aligned}
$$

Considering that $\frac{n^{2}+n}{2}=1+2+\cdots+n$ and looking just to the term inside the sum we can see that the coefficient of $t^{n}$ in that sum is the generating function for overpartitions where every (non-overlined) integer from one to the largest part appears at least once, the overlined parts are less than the largest non-overlined part and the number of parts is either $n$ or $n-1$. We call those overpartitions of type $n$.

Now, by making $q=1$ in (19), one can see that we get the sequence of the Pell numbers which allows us, taking into consideration the factor $1 /(1-t)$ outside the sum, to state the following theorem:

Theorem 3.1 The total number of overpartitions of type $n$ for all $n$ up to $N$ is equal to $p_{N}$.

In Table 1 we present the overpartitons for a few values of $N$. For example, in the third line, second column we have all overpartitions of type 3 , i.e., the ones with either 3 or 2 parts. 12 is the total up to that line.
Table 1. Partitions as in Theorem 3.1.

\begin{tabular}{lll}
\hline$N$ & \multicolumn{1}{c}{ Partitions } & $p_{N}$ \\
\hline 1 & $1 ; \varnothing$ & 2 \\
2 & $2+1 ; 1+1 ; 1$ & 5 \\
3 & $3+2+1 ; 2+2+1 ; \overline{1}+2+1$ & \\
& $2+1+1 ; 1+1+1 ;$ \\
& $2+1 ; 1+1$ \\
& $4+3+2+1 ; 3+3+2+1 ; 3+2+2+1 ;$ & \\
4 & $3+2+1+1 ; \overline{2}+3+2+1 ; \overline{1}+3+2+1 ;$ & \\
& $2+2+2+1 ; 2+2+1+1 ; 2+1+1+1 ;$ & \\
& $1+1+1+1 ; \overline{1}+2+1+1 ; \overline{1}+2+2+1 ;$ \\
& $3+2+1 ; 2+2+1 ; \overline{1}+2+1 ;$ \\
& $2+1+1 ; 1+1+1$ \\
\hline
\end{tabular}

For Equation (2) we have, with the parameter $t$ :

$$
\begin{aligned}
f_{16}(q, t) & =\sum_{n=0}^{\infty} \frac{t^{n} q^{n^{2}+2 n}}{\left(t ; q^{2}\right)_{n+1}\left(-t q^{2} ; q^{2}\right)_{n}} \\
& =\frac{1}{1-t} \sum_{n=0}^{\infty} \frac{t^{n} q^{n^{2}+2 n}}{\left(t^{2} q^{4} ; q^{4}\right)_{n}} .
\end{aligned}
$$

From this sum one can get

$$
(1-t)\left(1+t q^{2}\right) f_{16}(q, t)=1+t q^{2}+t q^{3} f_{16}\left(q, t q^{2}\right) .
$$

Knowing that the coefficient of $t^{n}$ in the expansion of (22) is a polynomial in $q$, i.e., that:

$$
f_{16}(q, t)=\sum_{n=0}^{\infty} P_{n} t^{n}
$$

we get:

$$
\begin{aligned}
& P_{0}(q)=1 ; P_{1}(q)=1+q^{3} ; \\
& P_{n}(q)=\left(1-q^{2}+q^{2 n+1}\right) P_{n-1}(q)+q^{2} P_{n-2}(q) .
\end{aligned}
$$

It is ease to see that at $q=1$ the sequence above is the Fibonacci sequence.

An explicity formula for this sequence is given bellow:

$$
\begin{aligned}
P_{n}(q) & =\sum_{j=-\infty}^{\infty} q^{10 j^{2}+3 j} U(2 n, 5 j) \\
& -\sum_{j=-\infty}^{\infty} q^{10 j^{2}+13 j+4} U(2 n, 5 j+3) .
\end{aligned}
$$

We explain now how to get a combinatorial interpretation for

$$
f_{16}(q, t)=\frac{1}{1-t} \sum_{n=0}^{\infty} \frac{t^{n} q^{n^{2}+2 n}}{\left(t^{2} q^{4} ; q^{4}\right)_{n}} .
$$


We consider the exponent $n(n+2)$ of $q$ as the sum $3+5+\cdots+2 n+1$ which means that the largest part is odd and every odd from 3 up to $2 n+1$ appears exactly once. In the denominator we will see a number of the form $4 n$ as $2 n+2 n$ which implies that each even number appears an even number of times. With these observations and taking into consideration the factor $1 /(1-t)$ we can state the following theorem.

Theorem 3.2 The total number of partitions into at most $N$ parts where the largest part is odd, every odd from 3 up to the largest part appears exactly once and each even part appears an even number of times is equal to $F_{\mathrm{N}+2}$.

Table 2 has, as an illustration, a few partitions as described in this theorem.

For identities 20, 28, 44, 66, 67, 80 and 81 we are going to list, for each one of them, the two variable function, the functional equation, the recurrence relation for the family of polynomials associated to it, the formula for this family and the corresponding theorem that it is possible to get with the combinatorial interpretation at $q=1$. A table to illustrate the result for small values of $n$ is also given.

\section{The Results from Equation (20)}

The two variable function:

$$
\begin{aligned}
f_{20}(q, t) & =\sum_{n=0}^{\infty} \frac{t^{n} q^{n^{2}}}{\left(t ; q^{2}\right)_{n+1}\left(-t q^{2} ; q^{2}\right)_{n}} \\
& =\frac{1}{1-t} \sum_{n=0}^{\infty} \frac{t^{n} q^{n^{2}}}{\left(t^{2} q^{4} ; q^{4}\right)_{n}} .
\end{aligned}
$$

Table 2. Partitions as in Theorem 3.2.

\begin{tabular}{lll}
\hline$N$ & \multicolumn{1}{c}{ Partitions } & $F_{N+2}$ \\
\hline 0 & $\varnothing$ & 1 \\
1 & 3 & 2 \\
2 & $3+5$ & 3 \\
3 & $7+5+3$ & \\
& $3+2+2$ & 5 \\
4 & $9+7+5+3$ & \\
& $5+4+4+3$ & \\
& $5+3+2+2$ & \\
5 & $11+9+7+5+3$ & \\
& $7+6+6+5+3$ & \\
& $7+5+3+4+4$ & \\
& $7+5+3+2+2$ & \\
& $3+2+2+2+2$ & \\
\hline
\end{tabular}

The functional equation:

$$
\begin{aligned}
& (1-t)\left(1+t q^{2}\right) f_{20}(q, t)=1+t q^{2}+t q f_{20}\left(q, t q^{2}\right), \\
& f_{20}(q, t)=\sum_{n=0}^{\infty} P_{n} t^{n} .
\end{aligned}
$$

The recurrence relation:

$$
\begin{aligned}
& P_{0}(q)=1 ; P_{1}(q)=1+q ; \\
& P_{n}(q)=\left(1-q^{2}+q^{2 n-1}\right) P_{n-1}(q)+q^{2} P_{n-2}(q) .
\end{aligned}
$$

An explicit formula for this family:

$$
\begin{aligned}
P_{n}(q) & =\sum_{j=-\infty}^{\infty} q^{10 j^{2}+j} U(2 n, 5 j) \\
& -\sum_{j=-\infty}^{\infty} q^{10 j^{2}+11 j+3} U(2 n, 5 j+2) .
\end{aligned}
$$

Here we will see a number of the form $4 n$ as $2 n+2 n$ as we have done for Equation (2).

Theorem 4.1 The total number of partitions into at most $N$ parts where every odd from one up to the largest appears exactly once, every even appears an even number of times and the largest even is at most one plus the largest odd is equal to $F_{N+1}$.

Table 3 has, as an illustration, a few partitions as described in this theorem.

\section{The Results from Equation (28)}

The two variable function:

$$
f_{28}(q, t)=\sum_{n=0}^{\infty} \frac{\left(-t q^{2} ; q^{2}\right)_{n} t^{n} q^{n^{2}+n}}{\left(t ; q^{2}\right)_{n+1}\left(-t q ; q^{2}\right)_{n+1}} .
$$

Table 3. Partitions as in Theorem 4.1.

\begin{tabular}{lll}
\hline$N$ & \multicolumn{1}{c}{ Partitions } & $F_{N+2}$ \\
\hline 0 & $\varnothing$ & 1 \\
1 & 1 & 2 \\
2 & $3+1$ & 3 \\
3 & $5+3+1$ & \\
& $2+2+1$ & 5 \\
4 & $7+5+3+1$ & \\
& $3+2+2+1$ & \\
& $4+4+3+1$ & \\
5 & $9+7+5+3+1$ & \\
& $2+2+2+2+1$ & \\
& $5+3+2+2+1$ & \\
& $5+4+4+3+1$ & \\
& $6+6+5+3+1$ & \\
\hline
\end{tabular}


The functional equation:

$$
\begin{aligned}
& (1-t)(1-t q) f_{28}(q, t)=1+\left(1+t q^{2}\right) t q^{2} f_{28}\left(q, t q^{2}\right), \\
& f_{28}(q, t)=\sum_{n=0}^{\infty} P_{n} t^{n} .
\end{aligned}
$$

The recurrence relation:

$$
\begin{aligned}
& P_{0}(q)=1 ; P_{1}(q)=1-q+q^{2} \\
& P_{n}(q)=\left(1-q+q^{2 n}\right) P_{n-1}(q)+\left(q+q^{2 n}\right) P_{n-2}(q)
\end{aligned}
$$

An explicit formula for this family:

$$
\begin{aligned}
P_{n}(q) & =\sum_{j=-\infty}^{\infty} q^{12 j^{2}+4 j} C T(2 n+1,1+6 j) \\
& +\sum_{j=-\infty}^{\infty} q^{12 j^{2}-8 j+1} C T(2 n+1,2-6 j) .
\end{aligned}
$$

Before stating the corresponding theorem we need a definition.

We call a green-yellow partition as the one where the parts may be of two colors, green or yellow, where the green parts are even and appear at least once and at most twice. For the yellow parts the only restriction is that the largest part is at most one plus twice the largest green part.

Theorem 5.1 The total number of green-yellow partitions into at most $N$ parts with an even number of odd parts minus the number of those having an odd number of odd parts is equal to $J_{N}$.

Table 4 has, as an illustration, a few partitions as described in this theorem.

\section{The Results from Equation (44)}

The two variable function:

Table 4. Partitions as described in Theorem 5.1.

\begin{tabular}{llll}
\hline \multicolumn{3}{c}{ Partitions } \\
\hline$N \quad$ Even number of odd parts & Odd number of odd parts & $J_{N}$ \\
0 & $\varnothing$ & 1 \\
1 & $2_{g}$ & 1 \\
& $2_{g}+2_{g} ; 2_{g}+2_{y} ;$ & 3 \\
2 & $1_{y}$ & \\
& $1_{y}+1_{y} ; 4_{g}+2_{g}$ & $2_{g}+1_{y} ; 2_{g}+3_{y}$ & \\
& $6_{g}+4_{g}+2_{g} ; 4_{g}+2_{g}+2_{g} ;$ & $4_{g}+2_{g}+1_{y} ; 4_{g}+2_{g}+3_{y} ;$ \\
& $4_{g}+2_{g}+2_{y} ;$ & $4_{g}+2_{g}+5_{y} ;$ \\
& $2_{g}+2_{g}+2_{y} ; 2_{g}+2_{y}+2_{y} ;$ & $2_{g}+2_{g}+1_{y} ; 2_{g}+2_{g}+3_{y} ;$ \\
& $2_{g}+1_{y}+1_{y} ;$ & $1_{y}+1_{y}+1_{y} ;$ \\
& $2_{g}+3_{y}+3_{y} ; 2_{g}+3_{y}+1_{y} ;$ & $2_{g}+3_{y}+2_{y} ; 2_{g}+2_{y}+1_{y}$ & \\
& $4_{g}+4_{g}+2_{g} ;$ & & 5 \\
& $4_{g}+2_{g}+4_{y}$ & &
\end{tabular}

$$
f_{44}(q, t)=\sum_{n=0}^{\infty} \frac{t^{3 n} q^{\frac{3}{2} n(n+1)}}{\left(t^{2} q ; q^{2}\right)_{n+1}(t ; q)_{n+1}} .
$$

The functional equation:

$$
\begin{aligned}
& (1-t)\left(1-t^{2} q\right) f_{44}(q, t)=1+t^{3} q^{3} f_{44}(q, t q), \\
& f_{44}(q, t)=\sum_{n=0}^{\infty} P_{n} t^{n} .
\end{aligned}
$$

The recurrence relation:

$$
\begin{aligned}
& P_{0}(q)=1 ; P_{1}(q)=1 ; P_{2}(q)=1+q ; \\
& P_{n}(q)=P_{n-1}(q)+q P_{n-2}(q)-\left(q-q^{n}\right) P_{n-3}(q) .
\end{aligned}
$$

An explicit formula for this family:

$$
\begin{aligned}
P_{n}(q) & =\sum_{j=-\infty}^{\infty} q^{80 j^{2}+12 j} 2 n+1 n-10 j \\
& -\sum_{j=-\infty}^{\infty} q^{80 j^{2}-28 j+2} 2 n+1 n-1+10 j \\
& -\sum_{j=-\infty}^{\infty} q^{80 j^{2}+52 j+8} 2 n+1 n-3-10 j \\
& +\sum_{j=-\infty}^{\infty} q^{80 j^{2}-68 j+14} 2 n+1 n-4+10 j .
\end{aligned}
$$

We need a definition before stating the next theorem.

We call a black-white partition as the one where the parts may be of two colors, black or white, each black part from one up to the largest part appears at least three times, each white part is odd and counted twice and the largest white part is at most one plus twice the largest black part.

Theorem 6.1 The total number of black-white partitions into at most $N$ parts is equal to $F_{N+1}$.

Table 5 has, as an illustration, a few partitions as described in this theorem.

\section{The Results from Equation (66)}

The two variable function:

$$
\begin{aligned}
f_{66}(q, t) & =\sum_{n=0}^{\infty} \frac{\left(-t^{2} ; q^{4}\right)_{n} t^{n} q^{n^{2}}}{\left(t ; q^{2}\right)_{n+1}\left(t q ; q^{2}\right)_{n}\left(-t q^{2} ; q^{2}\right)_{n}} \\
& =\frac{1}{1-t} \sum_{n=0}^{\infty} \frac{\left(1+t^{2}\right)\left(-t^{2} q^{4} ; q^{4}\right)_{n-1} t^{n} q^{n^{2}}}{\left(t q ; q^{2}\right)_{n}\left(t^{2} q^{4} ; q^{4}\right)_{n}} .
\end{aligned}
$$

The functional equation:

$$
\begin{aligned}
& (1-t)(1-t q)\left(1+t^{2} q\right) f_{66}(q, t) \\
& =(1-t q)\left(1+t q^{2}\right)+\left(1+t^{2}\right) t q f_{66}\left(q, t q^{2}\right), \\
& f_{66}(q, t)=\sum_{n=0}^{\infty} P_{n} t^{n}
\end{aligned}
$$


Table 5. Partitions as described in Theorem 6.1.

\begin{tabular}{cll}
\hline$N$ & \multicolumn{1}{c}{ Partitions } & $F_{N+1}$ \\
\hline 0 & $\varnothing$ & 1 \\
1 & & 1 \\
2 & $1_{w}$ & 2 \\
3 & $1_{b}+1_{b}+1_{b}$ & 3 \\
4 & $1_{w}+1_{w} ; 1_{b}+1_{b}+1_{b}+1_{b}$ & 5 \\
5 & $1_{b}+1_{b}+1_{b}+1_{b}+1_{b} ;$ & \\
& $1_{b}+1_{b}+1_{b}+1_{w} ;$ & \\
& $1_{b}+1_{b}+1_{b}+3_{w}$ & \\
& $1_{w}+1_{w}+1_{w} ;$ \\
& $1_{b}+1_{b}+1_{b}+1_{b}+1_{w} ;$ & \\
& $1_{b}+1_{b}+1_{b}+1_{b}+3_{w} ;$ & \\
& $2_{b}+2_{b}+2_{b}+1_{b}+1_{b}+1_{b} ;$ & \\
& $1_{b}+1_{b}+1_{b}+1_{b}+1_{b}+1_{b}$. & 13 \\
\hline
\end{tabular}

The recurrence relation:

$$
\begin{aligned}
P_{0}(q) & =1 ; P_{1}(q)=1+q ; P_{2}(q)=1+q+q^{2}+q^{4} ; \\
P_{n}(q)= & \left(1+q-q^{2}+q^{2 n-1}\right) P_{n-1}(q) \\
& -\left(q-q^{2}-q^{3}\right) P_{n-2}(q) \\
& -\left(q^{3}-q^{2 n-5}\right) P_{n-3}(q) .
\end{aligned}
$$

An explicit formula for this family:

$$
\begin{aligned}
P_{n}(q) & =\sum_{j=-\infty}^{\infty} q^{32 j^{2}+4 j} U(2 n-1,8 j) \\
& -\sum_{j=-\infty}^{\infty} q^{32 j^{2}-28 j+6} U(2 n+1,3-8 j) \\
& +q \sum_{j=-\infty}^{\infty} q^{32 j^{2}+12 j} U(2 n+1,1+8 j) \\
& -q \sum_{j=-\infty}^{\infty} q^{32 j^{2}-20 j+2} U(2 n-1,2-8 j) .
\end{aligned}
$$

We define a yellow-white partition as the one where the parts may be of two colors, yellow or white, where the white parts are odd and every odd from 1 up to the largest odd part $(2 n-1)$ appears at least once, the yellow parts are even, appearing in pairs, with the largest one being one plus the largest odd $(2 n)$ and for each pair of even up to the largest odd minus one there is a overlined part, i.e., for those parts (pairs of even from 2 up to $2 n-2$ ) actually we do have an overpartition.

Theorem 7.1 If we consider for each $n$ the yellowwhite partitions with $n$ or $n-2$ part then the total number of all such partitions with $n \leq N$ is equal to $Y_{N}$, where $Y_{N}$ denote the general term for sequence
A052542 from The On-Line Encyclopedia of Integer Sequences. This sequence, apart form the inicial 1, is simply twice the Pell numbers.

Table 6 has, as an illustration, a few partitions as described in this theorem.

\section{The Results from Equation (67)}

The two variable function:

$$
\begin{aligned}
f_{67}(q, t) & =\sum_{n=0}^{\infty} \frac{\left(-t^{2} ; q^{4}\right) t^{n} q^{n^{2}+2 n}}{\left(t ; q^{2}\right)_{n+1}\left(t q ; q^{2}\right)_{n}\left(-t q^{2} ; q^{2}\right)_{n}} \\
& =\frac{1}{1-t} \sum_{n=0}^{\infty} \frac{\left(1+t^{2}\right)\left(-t^{2} q^{4} ; q^{4}\right)_{n-1} t^{n} q^{n^{2}+2 n}}{\left(t q ; q^{2}\right)_{n}\left(t^{2} q^{4} ; q^{4}\right)_{n}} .
\end{aligned}
$$

The functional equation:

$$
\begin{aligned}
& (1-t)(1-t q)\left(1-t q^{2}\right) f_{67}(q, t) \\
& =(1-t q)\left(1+t q^{2}\right)-\left(1+t^{2}\right) t q^{3} f_{67}(q, t), \\
& f_{67}(q, t)=\sum_{n=0}^{\infty} P_{n} t^{n} .
\end{aligned}
$$

The recurrence relation:

$$
\begin{aligned}
P_{0}(q) & =1 ; P_{1}(q)=1+q^{3} ; P_{2}(q)=1+q^{3}+q^{4}+q^{8} \\
P_{n}(q) & =\left(1+q-q^{2}+q^{2 n+1}\right) P_{n-1}(q) \\
& -\left(q-q^{2}-q^{3}\right) P_{n-2}(q) \\
& -\left(q^{3}-q^{2 n-3}\right) P_{n-3}(q)
\end{aligned}
$$

An explicit formula for this family:

$$
\begin{aligned}
P_{n}(q) & =\sum_{j=-\infty}^{\infty} q^{32 j^{2}+4 j} U(2 n, 8 j) \\
& -\sum_{j=-\infty}^{\infty} q^{32 j^{2}-28 j+6} U(2 n-2,3-8 j) \\
& -q \sum_{j=-\infty}^{\infty} q^{32 j^{2}+12 j} U(2 n, 1+8 j) \\
& +q \sum_{j=-\infty}^{\infty} q^{32 j^{2}-20 j+2} U(2 n-2,2-8 j) .
\end{aligned}
$$

Table 6. Partitions as described in Theorem 7.1.

\begin{tabular}{lll}
\hline$N$ & \multicolumn{1}{c}{ Partitions } & $Y_{N}$ \\
\hline 0 & $\varnothing$ & 1 \\
1 & $1_{w}$ & 2 \\
2 & $3_{w}+1_{w} ; 1_{w}+1_{w}$ & 4 \\
3 & $5_{w}+3_{w}+1_{w} ; 3_{w}+1_{w}+1_{w} ;$ & \\
& $3_{w}+3_{w}+1_{w} ; 1_{w}+1_{w}+1_{w} ;$ & \\
& $1_{w}+2_{y}+2_{y} ; 1_{w}$ & 10 \\
\hline
\end{tabular}


We define a blue-red partition as the one where the parts may be of two colors, blue or red, where the red parts are odd, the largest red part $(2 n+1)$ appears only once and every odd from 3 up to the largest odd part $(2 n-1)$ appears at least once, the blue parts are even, appearing in pairs, with the largest blue part $(2 n)$ been one plus the largest odd and for each pair of even up to the largest odd minus one there is a overlined part, i.e., for those parts (pairs of even from 2 up to $2 n-2$ ) actually we do have an overpartition.

Theorem 8.1 If we consider for each $n$ the blue-red partitions with $n$ or $n-2$ parts then the total number of all such partitions with $n \leq N$ is equal to $B_{N}$, where $B_{N}$ denote the general term for sequence $A 052542$ from The On-Line Encyclopedia if Integer Sequences.

Table 7 has, as an illustration, a few partitions as described in this theorem.

\section{The Results from Equation (80)}

The two variable function:

$$
f_{80}(q, t)=\sum_{n=0}^{\infty} \frac{(-t ; q)_{n+1} t^{n} q^{\frac{1}{2} n(n+1)}}{\left(t^{2} ; q^{2}\right)_{n+1}\left(t^{2} q ; q^{2}\right)_{n+1}} .
$$

The functional equation:

$$
\begin{aligned}
& (1-t)\left(1-t^{2} q\right) f_{80}(q, t)=1+t q f_{80}(q, t q), \\
& f_{80}(q, t)=\sum_{n=0}^{\infty} P_{n} t^{n} .
\end{aligned}
$$

The recurrence relation:

$$
\begin{aligned}
& P_{0}(q)=1 ; P_{1}(q)=1+q ; P_{2}(q)=1+2 q+q^{2}+q^{3} ; \\
& P_{n}(q)=\left(1+q^{n}\right) P_{n-1}(q)+q P_{n-2}-q P_{n-3}(q) .
\end{aligned}
$$

An explicit formula for this family:

$$
\begin{aligned}
P_{n}(q) & =\sum_{j=-\infty}^{\infty} q^{42 j^{2}+5 j} C T(n, 1+14 j) \\
& +\sum_{j=-\infty}^{\infty} q^{42 j^{2}-37 j+8} C T(n, 6-14 j) \\
& -q^{2}\left(\sum_{j=-\infty}^{\infty} q^{42 j^{2}+19 j} C T(n, 3+14 j)\right. \\
& \left.+\sum_{j=-\infty}^{\infty} q^{42 j^{2}-23 j+1} C T(n, 4-14 j)\right) .
\end{aligned}
$$

We need a definition before stating the next theorem.

We call a green-black partition as the one where the parts may be of two colors, green or black, each green part from one up to the largest part appears at least once and at most twice, each black part is counted twice and the largest black part is at most one plus twice the largest green part.
Theorem 9.1 The total number of green-black partitions into at most $N$ parts is equal to $G_{N}$, where $G_{N}$ denote the general term for sequence A006054 from The On-Line Encyclopedia if Integer Sequences.

Table 8 has, as an illustration, a few partitions as described in this theorem.

\section{The Results from Equation (81)}

The two variable function:

$$
f_{81}(q, t)=\sum_{n=0}^{\infty} \frac{(-t ; q)_{n+1} t^{n} q^{\frac{1}{2} n(n+1)}}{\left(t^{2} ; q^{2}\right)_{n+1}\left(t^{2} q ; q^{2}\right)_{n}} .
$$

The functional equation:

$$
\begin{aligned}
& (1-t)\left(1-t^{2} q\right) f_{81}(q, t)=1-t^{2} q+t q f_{81}(q, t q), \\
& f_{81}(q, t)=\sum_{n=0}^{\infty} P_{n} t^{n} .
\end{aligned}
$$

The recurrence relation:

$$
\begin{aligned}
& P_{0}(q)=1 ; P_{1}(q)=1+q ; P_{2}(q)=1+q+q^{2}+q^{3} ; \\
& P_{n}(q)=\left(1+q^{n}\right) P_{n-1}(q)+q P_{n-2}(q)-q P_{n-3}(q) .
\end{aligned}
$$

An explicit formula for this family:

Table 7. Partitions as described in Theorem 8.1.

\begin{tabular}{lll}
\hline$N$ & Partitions & $B_{N}$ \\
\hline 0 & $\varnothing$ & 1 \\
1 & $1_{r}$ & 2 \\
2 & $3_{r}+1_{r} ; 1_{r}+1_{r} ;$ & 4 \\
3 & $5_{r}+3_{r}+1_{r} ; 3_{r}+3_{r}+1_{r} ;$ & \\
& $3_{r}+1_{r}+1_{r} ; 1_{r}+1_{r}+1_{r} ;$ & \\
& $1_{r}+2_{b}+2_{b} ; 1_{r}$ & 10 \\
\hline
\end{tabular}

Table 8. Partitions as described in Theorem 9.1.

\begin{tabular}{lll}
\hline$N$ & \multicolumn{1}{c}{ Partitions } & $G_{N}$ \\
\hline 0 & $\varnothing$ & 1 \\
1 & $1_{g}$ & 2 \\
2 & $1_{b} ; 1_{g}+1_{g} ; 2_{g}+2_{g}$ & 5 \\
3 & $1_{g}+1_{b} ; 1_{g}+2_{b} ;$ & \\
& $1_{g}+3_{b} ; 2_{g}+2_{g}+1_{g} ;$ & \\
& $2_{g}+1_{g}+1_{g} ; 3_{g}+2_{g}+1_{g}$ & 11 \\
\hline
\end{tabular}




$$
\begin{aligned}
P_{n}(q) & =\sum_{j=-\infty}^{\infty} q^{42 j^{2}+j} C T(n, 14 j) \\
& +\sum_{j=-\infty}^{\infty} q^{42 j^{2}-41 j+10} C T(n, 7-14 j) \\
& -q\left(\sum_{j=-\infty}^{\infty} q^{42 j^{2}+13 j} C T(n, 2+14 j)\right. \\
& \left.-\sum_{j=-\infty}^{\infty} q^{42 j^{2}-29 j+4} C T(n, 5-14 j)\right) .
\end{aligned}
$$

We need a definition before stating the next theorem.

We call a red-black partition as the one where the parts may be of two colors, red or black, each red part from one up to the largest part appears at least once and at most twice, each black part is counted twice and the largest black part is at most twice the largest red part.

Theorem 10.1 The total number of red-black partitions into at most $N$ parts is equal to $G_{N}$, where $G_{N}$ denote the general term for sequence A052534 from The On-Line Encyclopedia of Integer Sequences.

Table 9 has, as an illustration, a few partitions as described in this theorem.

Table 9. Partitions as described in Theorem 10.1.

\begin{tabular}{llc}
\hline$N$ & Partitions as described in the theorem \\
\hline 0 & $\varnothing$ & $G_{N}$ \\
1 & $1_{r}$ & 1 \\
2 & $2_{r}+1_{r} ; 1_{r}+1_{r}$ & 2 \\
3 & $3_{r}+2_{r}+1_{r} ; 2_{r}+2_{r}+1_{r} ;$ & 4 \\
& $2_{r}+1_{r}+1_{r} ; 1_{r}+1_{b} ;$ \\
& $1_{r}+2_{b}$ \\
& $1_{r}+1_{r}+1_{b} ; 2_{r}+1_{r}+1_{b} ;$ \\
& $2_{r}+1_{r}+2_{b}, 2_{r}+1_{r}+3_{b} ;$ \\
& $1_{r}+1_{r}+2_{b} ; 2_{r}+1_{r}+4_{b} ;$ \\
& $3_{r}+2_{r}+1_{r}+1_{r} ; 3_{r}+2_{r}+2_{r}+1_{r} ;$ \\
& \\
& $3_{r}+3_{r}+2_{r}+1_{r}$ \\
& $4_{r}+3_{r}+2_{r}+1_{r} ; 2_{r}+2_{r}+1_{r}+1_{r}$ \\
\end{tabular}

\section{Conclusion}

It is our believe that more results of similar type may be obtained following the ideas used in this paper. The computer algebra packages available today are a powerful tool in the study of q-series identities of the RogersRamanujan type.

\section{REFERENCES}

[1] J. P. O. Santos and M. Ivkovic, "Polynomial Generalizations of the Pell Sequence and the Fibonacci Sequence," The Fibonacci Quarterly, Vol. 43, No. 4, 2005, pp. 328338.

[2] J. P. O. Santos and M. Ivkovic, "Fibonacci Numbers and Partitions," The Fibonacci Quarterly, Vol. 41, No. 3, 2003, pp. 263-278.

[3] J. P. O. Santos, "On the Combinatorics of Polynomial Generalizations of Rogers-Ramanujan-Type Identities," Discrete Mathematics, Vol. 254, No. 1-3, 2002, pp. 497 511. doi:10.1016/S0012-365X(01)00378-8

[4] G. E. Andrews, "Combinatorics and Ramanujan's 'Lost' Notebook," In: London Mathematical Society Lecture Note Series, No. 103, Cambridge University Press, London, 1985, pp. 1-23.

[5] J. P. O. Santos, "Computer Algebra and Identities of the Rogers-Ramanujan Type,” Ph.D. Thesis, Pennsylvania State University, University Park, 1991.

[6] L. J. Slater, "Further Identities of the Rogers-Ramanujan Type," Proceedings London Mathematical Society, Vol. s2-54, No. 1, 1952, pp. 147-167. doi: $10.1112 / \mathrm{plms} / \mathrm{s} 2-54.2 .147$

[7] L. J. Slater, "A New Proof of Roger's Transformations of Infinite Series," Proceedings London Mathematical Society, Vol. s2-53, No. 1, 1951, pp. 460-475. doi: $10.1112 / \mathrm{plms} / \mathrm{s} 2-53.6 .460$

[8] A. V. Sills. "RRtools-A Maple Package for Aiding the Discovery and Proof of Finite Rogers-Ramanujan Type Identities," Journal of Symbolic Computation, Vol. 37, No. 4, 2004, pp. 415-448.

http://math.georgiasouthern.edu/asills/maple/RRtools1 